\title{
Calibration of Magnetometer for Small Satellites Using Neural Network
}

\author{
T. Kliment ${ }^{a, *}$, D. PrasličKa ${ }^{a}$, P. LipovskÝ ${ }^{a}$, K. Draganová ${ }^{a}$ And O. ZÁvodský ${ }^{b, c}$ \\ ${ }^{a}$ Department of Aviation Technical Studies, Faculty of Aeronautics, Technical University of Košice, \\ Rampová 7, 04121 Košice, Slovakia \\ ${ }^{b}$ Slovak Organization for Space Activities (SOSA), Zámocká 18, 81101 Bratislava, Slovakia \\ ${ }^{c}$ Department of Telecommunications and Multimedia, Faculty of Electrical Engineering, University of Žilina, \\ Univerzitná 1, 01026 Žilina, Slovakia
}

\begin{abstract}
The article presents the scalar calibration method that uses a neural network for the determination of parameters of the inverse model of the vector magnetometer. Utilization of the one layered, feed-forward neural network with the back propagation algorithm has suppressed the systematic errors of the vector magnetometers, namely the multiplicative, additive, orthogonality and linearity errors. Methodology shown in the article was designed and used for a pre-flight calibration of the magnetometer used in the first Slovak satellite skCUBE, where the magnetometer performs stabilization and navigation tasks. The experiment was performed in a 3D Helmholtz coil system, where the Earth magnetic field was suppressed and at the same time the stimulation field was created. Suppression of the Earth magnetic field was achieved by special positioning of the satellite. Honeywell HMC 5883L was used for the verification of the methodology.
\end{abstract}

DOI: 10.12693/APhysPolA.131.1129

PACS/topics: 06.20.fb

\section{Introduction}

In general, the main goal of the calibration methodologies is to improve sensor precision [1-3]. The calibration is particularly important if high precision of the miniature low-cost sensors is required. The article describes a scalar position-independent calibration methodology of $3 \mathrm{D}$ vector sensors, which decreases demands on the calibration hardware and thanks to the neural networks utilization it is possible to process datasets of any robustness. The calibration procedure was applied to the HMC 5883L magnetometer, which is a part of the first Slovak satellite of the CubeSat category shown in Fig. 1.

It is a satellite with the dimensions of $10 \times 10 \times 10 \mathrm{~cm}^{3}$ and with the weight up to the $1.33 \mathrm{~kg}$, which is desig-

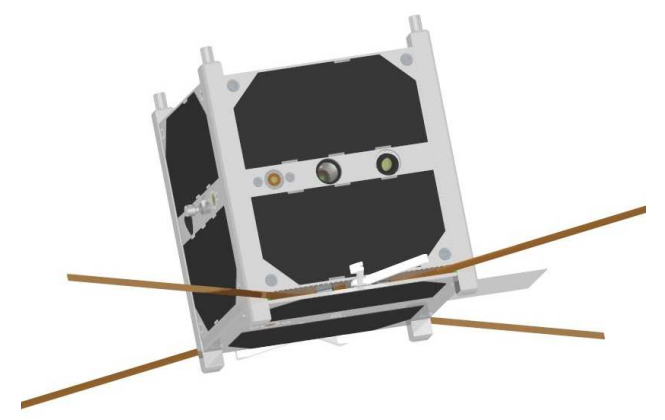

Fig. 1. The CAD model of the skCUBE.

*corresponding author; e-mail: tomas.kliment@tuke.sk nated as the " $1 U$ " standard. The magnetometer fulfills navigational and stabilization tasks that are important for stopping of the rotation, which can be present after the satellite launch from the carrying missile container, applying the B-DOT algorithm [4].

\section{Theory}

The dominant systematic errors of the vector sensors are multiplicative, additive, linearity and orthogonality errors. The fundamental inverse model, in which the mentioned errors are included, can be defined for each channel as:

$$
\begin{aligned}
& \tilde{x}=C_{x} x^{3}+B_{x} x+A_{x}, \\
& \tilde{y}=C_{y} y^{3}+B_{y} y+A_{y}+O_{y x} x, \\
& \tilde{z}=C_{z} z^{3}+B_{z} z+A_{z}+O_{z y} y+O_{z x} x,
\end{aligned}
$$

where $x, y$ and $z$ are sensor outputs representing the uncalibrated orthogonal decomposition of the normalized vector, $\tilde{x}, \tilde{y}$ and $\tilde{z}$ are corrected values, $C$ are linearity calibration constants, $B-$ multiplicative constants, $A$ - additive constants and $O$ - orthogonality constants. The goal of the calibration procedure is to find these calibration constants using the one-layer neural network working above the set of input vectors represented by the measured $x, y$ and $z$ values of the decomposition. Similar principle is discussed in [5], where a method working in real time is used, but the method has worse quality of convergence. For example it is not possible to decorrelate the data, to random ordering of the samples or to center the data. The iteration procedure uses the backpropagation algorithm [6] based on the gradient descent 
methodology, in which the error function $E$ in the $k$-th step of the learning process is

$$
E^{k}=\frac{1}{2}\left(T^{k}-1\right)^{2},
$$

where $T$ is a normalized module of the magnetic induction vector

$$
T^{k}=\sqrt{\left(\tilde{x}^{k}\right)^{2}+\left(\tilde{y}^{k}\right)^{2}+\left(\tilde{z}^{k}\right)^{2}} \approx 1 .
$$

In the beginning the calibration constants are implicitly set to the following values: $C=0, B=1, A=0$ and $O=0$. Increments of each constant in the $k$-th step of the learning process are in general defined as:

$$
\Delta K_{i}^{k}=-\left.\alpha \frac{\partial E}{\partial K_{i}}\right|_{k},
$$

where $\alpha$ represents velocity and stability of the learning process. In our case it was set to 0.01 .

\section{Experiment}

The experiment was performed in the Slovak Organization for Space Activities (SOSA) laboratory in Bratislava. For the calibration a three-axial system of the Helmholtz coils with the sensitivities experimentally determined according to the [7] was used. The precision of the generated field was $\pm 0.1 \mu \mathrm{T}$. The main task of this coil system is to create $n$ vectors, the attractors of which created points equally distributed on the sphere surface to obtain the constant magnetic induction module of $60 \mu \mathrm{T}$. The Earth magnetic field was suppressed in several steps.

In the first one the Earth magnetic field components using the vector magnetic analyzer (VEMA) relaxation magnetometer [8] positioned into the two axes of the coils applying of the current reversing in the Helmholtz coils were determined. Subsequently vectors corrected in regard to the measured Earth magnetic field using the appropriate methodology were generated. The data were uniformly distributed on the surface of a sphere. The principle is based on horizontal slicing of the sphere, so each slice has the same area. One point is placed on each sliced sphere surface, so they do not create clusters. These fields representing the first dataset with 200 vectors of the field were measured by the satellite magnetometer. In the second step we turned the satellite magnetometer by $180^{\circ}$ around the $z$ axis and flipped the excitation currents in the $x$ and $y$ axes. The second dataset containing 200 vectors was then obtained. In the third step the procedure was repeated for the magnetometer turn around the $y$ axis in regard to the initial position and the directions of the excitation currents in the $x$ and $z$ axes were flipped. From these 3 positions one file comprising averaged values representing the training set for the neural network with suppressed the influence of the Earth magnetic field as a residuum resulting from the vector VEMA magnetometer inaccuracies was created. Without the precise mechanical positioning of the satellite it is not possible to suppress the remaining Earth magnetic field components. The measured data were normalized, sequentially averaged over
4 samples and randomly reordered. Finally the learning process using Eqs. (2.1)-(2.4) on the training set was repeatedly performed. The comparison of the normalized linear errors during the calibration procedure is visualized in Fig. 2.

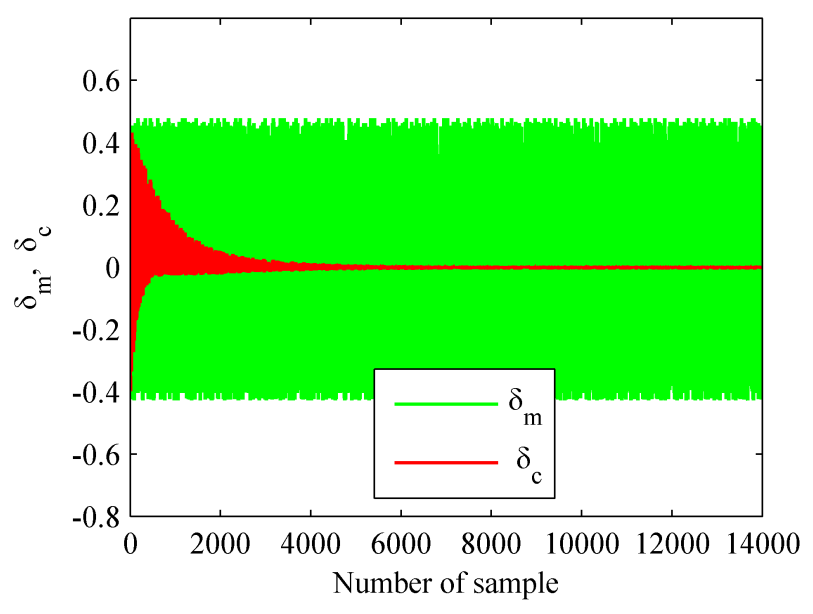

Fig. 2. Comparison of the normalized linear errors calculated from the uncalibrated data $\left(\delta_{m}\right)$ and data corrected during the learning process $\left(\delta_{c}\right)$.

The normalized linear error $\delta$ is defined as:

$$
\delta^{k}=T^{k}-1 \text {. }
$$

Values of the normalized calibration constants after the convergence achievement are summarized in Table I.

TABLE I

Overview of the determined normalized calibration constants.

\begin{tabular}{c|c|c|c|c}
\hline \hline \multirow{2}{*}{ Channel } & \multicolumn{4}{|c}{ Calibration constant } \\
\cline { 2 - 5 } & $C$ & $B$ & $A$ & $O$ \\
\hline$X$ & 0.0020 & 1.0193 & -0.4535 & - \\
$Y$ & 0.0015 & 1.1066 & -0.0300 & $0.0992\left(O_{y x}\right)$ \\
$Z$ & 0.0079 & 1.1037 & 0.0641 & $-0.0118\left(O_{z y}\right)$, \\
& & & & $-0.0165\left(O_{z x}\right)$
\end{tabular}

The experimentally determined normalized linear error of the non-calibrated sensor varies from -0.4267 to 0.4780, which represents the absolute peak-to-peak error of $54.279 \mu \mathrm{T}$. After the calibration process the error varies in the range from -0.0060 to 0.0045 representing the peak to peak value of $0.630 \mu \mathrm{T}$. The standard deviation of the scalar $T$ value before the calibration was $15.342 \mu \mathrm{T}$ and after the calibration process it was reduced to $0.103 \mu \mathrm{T}$. The residual error of the calibrated sensor has its origin probably in the internal sensor noise. The noise standard deviation $\sigma_{N}$ of the sensor is according to the datasheet $0.15 \mu \mathrm{T}$ for each channel. After the data averaging through the 4 samples the noise standard deviation is suppressed according to the relationship 


$$
\sigma_{N}=\frac{0.15}{\sqrt{4}}=0.075[\mu \mathrm{T}] .
$$

Considering the same noise in all three axes, the scalar value of the noise can be calculated as

$$
\sigma_{S}=\sqrt{\sigma_{N}^{2}+\sigma_{N}^{2}+\sigma_{N}^{2}}=0.130[\mu \mathrm{T}] .
$$

In regard to the statistical character of the parameters listed in the datasheets it can be concluded that the error after calibration approximates to its limiting value represented by the inherent noise of the sensor. In Fig. 3 measured and in Fig. 4 calibrated data on the sphere surface are visualized.

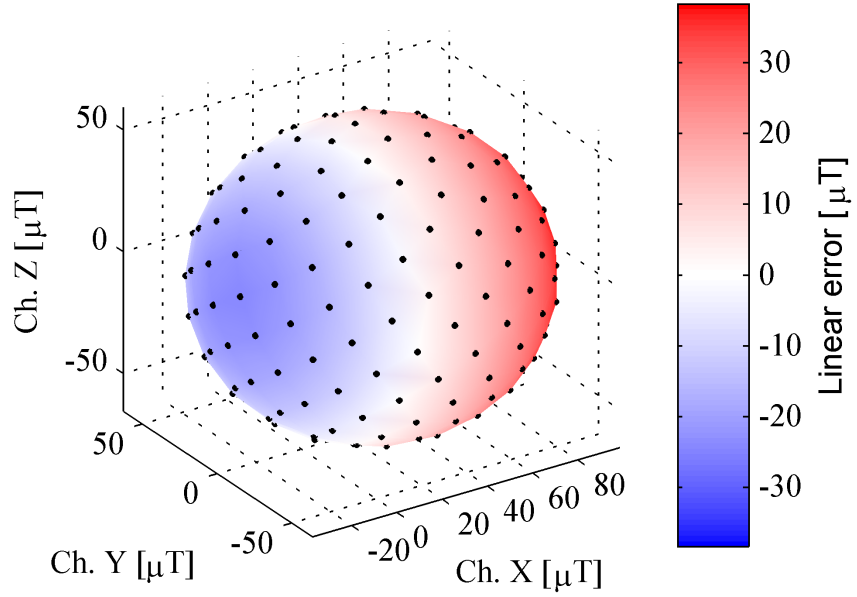

Fig. 3. A global map of errors of the uncalibrated sensor.

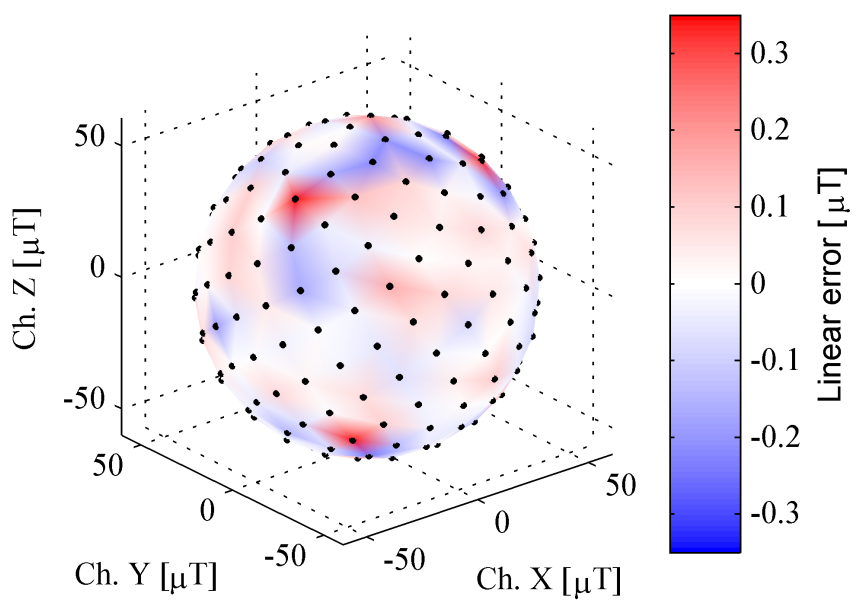

Fig. 4. A global map of errors of the calibrated sensor.

\section{Conclusions}

From the performed experiment results it can be stated that the precision of the non-calibrated HMC 5883L sensor is as big as $71.9^{\circ}$ in the heading, mainly due to the additive errors. The same sensor with the additive compensation achieves the precision of $6.0^{\circ}$ and after the application of all calibration constants to the inverse model the heading can be determined with the precision of $2.9^{\circ}$ in the worst case. The experiment confirmed that neural networks are convenient for the calibration of the on board vector magnetometer of the small satellite. After the calibration process the sensor performance is influenced only by the random errors with the statistical character. This fact was confirmed also by the random placement of the calibrated sensor errors on the sphere surface. It can be concluded that the inverse model was chosen correctly because the systematic errors were suppressed significantly.

\section{Acknowledgments}

This work was supported by the APVV 0266-10 and VEGA $1 / 0201 / 16$ projects.

\section{References}

[1] N. Olsen, L. Toffner-Clausen, T.J. Sabaka, P. Brauer, J.M.G. Merayo, J.L. Jorgensen, J.-M. Léger, O.V. Nielsen, F. Primdahl, T. Risbo, Earth Planets Space 55, 11 (2003).

[2] J.C. Springmann, J.W. Cutler, Acta Astronaut. 99, 201 (2014).

[3] J.L. Crassidis, K.-L. Lai, R.R. Harman, J. Guid. Control Dyn. 28, 115 (2005).

[4] M. Lovera, Proc. Am. Control Conf. 2015-July, 1867 (2015).

[5] T. Kliment, D. Praslicka, K. Draganova, J. Blazek, J. Electr. Eng. 66, 157 (2015).

[6] R. Rojas, Neural Networks, Springer, Berlin 1996.

[7] A. Zikmund, P. Ripka, J. Electr. Eng. 61, 39 (2013).

[8] J. Blazek, J. Hudak, D. Praslicka, Sens. Actuat. A Phys. 59, 287 (1997). 